\title{
Plaque Forming Unit
}

National Cancer Institute

\section{Source}

National Cancer Institute. Plaque Forming Unit. NCI Thesaurus. Code C67264.

A measure of viable infectious entities (e.g. viral particles or group of particles) in the specimen or product defined as the smallest quantity that can produce a cytopathic effect in the host cell culture challenged with the defined inoculum, visible under the microscope and/or to the naked eye as a plaque. A number of plaque forming units (PFU) per unit volume is a conventional way to refer the titer of an infective entity in a specimen or preparation. 